\title{
Inhibition of the Ras-ERK pathway in mitotic COS7 cells is due to the inability of EGFR/Raf to transduce EGF signaling to downstream proteins
}

\author{
HUAIPING SHI, TIANYING ZHANG, YONGQING YI and YUE MA \\ College of Animal Science and Technology, Northwest A\&F University, Yangling, Shaanxi 712100, P.R. China
}

Received November 6, 2015; Accepted February 3, 2016

DOI: $10.3892 /$ or.2016.4696

\begin{abstract}
Although previous studies have shown that Ras-ERK signaling in mitosis is closed due to the inhibition of signal transduction, the events involved in the molecular mechanisms are still unclear. In the present study, we investigated the Ras-ERK signaling pathway in mitotic COS7 cells. The results demonstrated that treatment with epidermal growth factor (EGF) failed to increase the endocytosis of EGF-EGFR (EGF receptor) complexes in mitotic COS7 cells, although a large amount of endosomes were found in asynchronous COS7 cells. Clathrin expression levels in mitotic COS7 cells were inhibited whereas caveolin expression levels in mitotic COS7 cells were almost unaffected. Y1068 and Y1086 residues of EGFR in the mitotic COS7 cells were activated. However, Grb2 and Shc in the mitotic COS7 cells did not bind to activated EGFR. Ras activity was inhibited in the mitotic COS7 cells whereas its downstream protein, Raf, was obviously phosphorylated by EGF in mitosis. Treatment with phorbol 12-myristate 13-acetate (PMA) also increased the phosphorylation levels of Raf in the mitotic COS7 cells. Nevertheless, Raf phosphorylation in mitosis was significantly inhibited by AG1478. Lastly, activation of EGF-mediated MEK and ERK in the mitotic COS7 cells was obviously inhibited. In summary, our results suggest that the Ras-ERK pathway is inhibited in mitotic COS7 cells which may be the dual result of the difficulty in the transduction of EGF signaling by EGFR or Raf to downstream proteins.
\end{abstract}

\section{Introduction}

The Ras-ERK signaling pathway mediates numerous cellular functions such as cell proliferation, differentiation, transformation and survival in different tissues and cell

Correspondence to: Dr Huaiping Shi, College of Animal Science and Technology, Northwest A\&F University, Xinong Road 22, Yangling, Shaanxi 712100, P.R. China

E-mail: huaipingshi@nwsuaf.edu.cn

Key words: EGF, Ras-ERK pathway, mitosis, COS7 cells types (1-4). Epidermal growth factor (EGF)-stimulated Ras activation is mediated by the adaptor protein Grb2, which can associate with activated EGFR directly via Y1068 and Y1086 sites, or indirectly through tyrosine phosphorylated Shc $(5,6)$. Through binding to Grb2, the protein Sos, a guanine nucleotide exchange factor (GEF) of Ras, relocates to EGFR at the plasma membrane and activates membrane-associated Ras, which in turn activates the subsequent Raf-MEK-ERK signaling cascade $(7,8)$. Finally, ERK translocates to the nucleus and activates several transcriptional factors such as c-Fos and c-Jun, driving cell proliferation and other processes $(9,10)$.

In eukaryotes, the standard cell cycle which is regulated by a series of molecular events is often divided into two periods: the interphase period and mitosis. At the cellular interphase, signaling from the Ras-ERK pathway facilitates progression through G1/S phase and through processes involved in nuclear transcription factor phosphorylation, immediate-early gene induction, expression of cell cycle genes that direct DNA synthesis and regulation of translational initiation $(11,12)$. Nevertheless, one of the marked changes in mitosis is general inhibition of membrane traffic. Accumulated studies have shown that membrane traffic is inhibited during mitosis and mitotic cells likely fail to respond to transmembrane signaling (13-17). The events during mitosis possibly preserve the high energy requirements needed for the dynamic structural changes that are occurring at this time of the cell cycle.

Previous research has found that some signaling pathways such as ERK signaling, are affected in mitosis $(18,19)$. Mitotic cells are less responsive to extracellular growth factor stimulation as compared with interphase cells. Due to reduced EGF and inhibition of EGF receptor dimerization, EGFR activity by EGF is reduced in mitosis $(20,21)$ and in turn affects the ERK signal transduction pathway. In addition, some Ras-ERK pathway proteins may undergo unique regulation during mitosis. Phosphorylated MEK-1 regulates partial proteolysis at the $\mathrm{N}$ terminus in mitosis, which results in the inability for MEK-1 to interact with and activate ERK1/2 proteins. However, it is unlikely that MEK1 is completely uncoupled from ERK1/2 during mitosis since activation of protein kinase $\mathrm{C}$ by treatment with phorbol esters can still activate the Raf-1/MEK/ERK pathway in mitotic cells (22). Notably, Raf-1 was activated in cells arrested in mitosis with nocodazole (23). Although the research demonstrated that Raf-1 activity is related to nocodazole stimulation, the regulatory mechanism 
is unclear at present, and the Raf function in mitosis remains unknown. As a Raf upstream protein, Ras activity in mitosis has not been investigated in detail.

The present study aimed to investigate the Ras-ERK signaling pathway in mitotic COS7 cells. Our findings indicate that the activities of Ras-ERK pathway proteins are almost blocked in mitosis, except for Raf protein. In addition, the ability of Grb2/Shc binding to activate EGFR was reduced. Thus, the inhibition of the Ras-ERK pathway in mitotic COS7 cells may be the dual results of the difficulty in the the transduction of EGF signaling by EGFR or Raf to downstream proteins.

\section{Materials and methods}

Cell culture. COS7 cells were grown at $37^{\circ} \mathrm{C}$ in Dulbecco's modified Eagle's medium (DMEM) containing 5\% fetal bovine serum (FBS), penicillin and streptomycin $(100 \mathrm{U} / \mathrm{ml})$ and were maintained in a $5 \% \mathrm{CO}_{2}$ atmosphere.

Antibodies and chemicals. Antibodies specific for phospho-ERK1/2 (E-4), tubulin, phospho-MEK1/2 (ser218/ ser222), pY1068-EGFR, pY1086-EGFR, Grb2 (C-23), Shc (PG-797), GST (B-14) and phospho-Raf (Tyr340/341) were purchased from Santa Cruz Biotechnology (Santa Cruz, CA, USA). An antibody specific for Ras was purchased from Upstate Biotechnology Inc. (Lake Placid, NY, USA). HRP-conjugated secondary antibodies were purchased from Bio-Rad (Hercules, CA, USA). Nocodazole and AG1478 were purchased from Calbiochem (La Jolla, CA, USA). EGF and phorbol 12-myristate 13-acetate (PMA) were purchased from Sigma (St. Louis, MO, USA). Unless otherwise specified, all of the chemicals were purchased from Sigma.

Cell treatment. To detect protein activity in mitosis, COS7 cells were treated with $200 \mathrm{ng} / \mathrm{ml}$ nocodazole for $24 \mathrm{~h}$, and then stimulated with or without $50 \mathrm{ng} / \mathrm{ml}$ EGF or $1 \mu \mathrm{M}$ PMA for the indicated time $(5,15$ and $30 \mathrm{~min})$.

To further investigate Raf activity in response to EGF, COS7 cells were pretreated with $0.5 \mu \mathrm{mol}$ AG1478 for $30 \mathrm{~min}$, and then stimulated with or without $50 \mathrm{ng} / \mathrm{ml} \mathrm{EGF}$ for $15 \mathrm{~min}$ in the continuous presence of the inhibitor.

Fluorescence microscopy. COS7 cells grown on glass coverslips were treated with or without $200 \mathrm{ng} / \mathrm{ml}$ nocodazole for $24 \mathrm{~h}$ and then treated with $50 \mathrm{ng} / \mathrm{ml}$ Texas Red (TR)-EGF for the indicated time $(5,15$ and $30 \mathrm{~min})$. After that, COS7 cells were fixed with icy methanol for $10 \mathrm{~min}$ followed by analysis and imaging with a Zeiss Axiovert 200 microscope (Carl Zeiss, Thornwood, NY, USA) and an AttoArc2 HBO 100W light source (Atto Instruments, Rockville, MD, USA).

Immunoprecipitation. COS7 cells were lysed with immunoprecipitation buffer overnight at $4^{\circ} \mathrm{C}$. COS7 cell lysates were then centrifuged at $21,000 \mathrm{x} \mathrm{g}$ for $30 \mathrm{~min}$ to remove debris. The supernatants, containing $1 \mathrm{mg}$ of total protein, were incubated with $1 \mu \mathrm{g}$ of mouse anti-EGFR antibody to immunoprecipitate EGFR from the COS7 cells. For the control experiments, primary antibodies were replaced with normal mouse or sheep IgG (Sigma), and no EGFR was precipitated by normal $\mathrm{IgG}$.
Ras activation assay. Ras activation was assayed by a method described by Herrmann et al (24). Briefly, COS7 cells which had been treated as required were lysed and scraped into $0.5 \mathrm{ml}$ of BOS buffer [50 mM Tris- $\mathrm{HCl}$ (pH 7.4), $200 \mathrm{mM} \mathrm{NaCl}, 1 \%$ NP-40, $10 \%$ glycerol, $10 \mathrm{mM} \mathrm{NaF,} 2.5 \mathrm{mM} \mathrm{MgCl} \mathrm{m}_{2}, 1 \mathrm{mM}$ EDTA), and then centrifuged at $21,000 \mathrm{x} \mathrm{g}$ and $4^{\circ} \mathrm{C}$ for $30 \mathrm{~min}$. Glutathione $S$-transferase (GST) fused to the Raf binding domain (GST-RBD), pre-coupled to glutathione-agarose beads in BOS buffer, was added, and the lysates were incubated at $4^{\circ} \mathrm{C}$ for $1 \mathrm{~h}$. The beads were collected by centrifugation and washed three times with BOS buffer, and then loading buffer was added. Ras was detected with the monoclonal anti-Ras antibody, followed by a horseradish peroxidase (HRP)-coupled anti-mouse antibody.

Immunoblotting. For the detection of phospho-EGFR, phospho-Ras, phospho-Raf, phospho-MEK, phospho-ERK, clathrin and caveolin in total lysates of COS7 cells, aliquots containing $20 \mu \mathrm{g}$ of protein from each cell lysate were used. For the detection of EGFR, Shc and Grb2 in the anti-EGFR immunoprecipitates, $1 / 10$ of the immunoprecipitate from each lysate was used. Protein samples were separated by electrophoresis through sodium dodecyl sulfate 10-7.5\% polyacrylamide-containing gels and electrophoretically transferred onto nitrocellulose filter paper. Filters were then probed with the respective primary antibody. The primary antibodies were detected with a polyclonal goat anti-rabbit IgG coupled to HRP or a polyclonal goat anti-mouse IgG coupled to HRP followed by enhanced chemiluminescence development (Pierce Chemical, Rockford, IL, USA) and light detection with Bioshine Chemi Q 2550.

Statistical analysis. In all cases, the data from three independent experiments were evaluated. The data of the western blot analyses are expressed as means \pm SE of triplicate measurements. All data were analyzed with the software package SPSS 19.0. Significance was declared at $\mathrm{P}<0.05$.

\section{Results}

Endocytosis of EGF-EGFR during mitosis. It has been shown that EGF induces dimerization of EGF receptor protein in intact cells $(25,26)$. After dimerization of EGFR, they form complexes and the latter in turn are endocytosed. In the present study, we used TR-EGF to treat COS7 cells in response to nocodazole and observed the endosomes in the cytoplasm. We found that the endosomes in the cytoplasm were observed after 5 min of TR-EGF stimulation and more with extension of time in the nocodazole-untreated COS7 cells. Nevertheless, only a few endosomes were detected after 15 min of TR-EGF stimulation in the nocodazole-treated COS7 cells and others were blocked in the plasma membrane (Fig. 1). In addition, the endosomes in the nocodazole-untreated COS7 cells were obviously more than that in the nocodazole-treated COS7 cells.

Receptor-mediated endocytosis consists of two pathways, clathrin-dependent and clathrin-independent endocytosis. The two pathways are detected by analyzing marker proteins, clathrin and caveolin. We found that clathrin expression levels were almost not upregulated after mitotic COS7 cells were stimulated for 30 min by EGF, although the levels of clathrin 

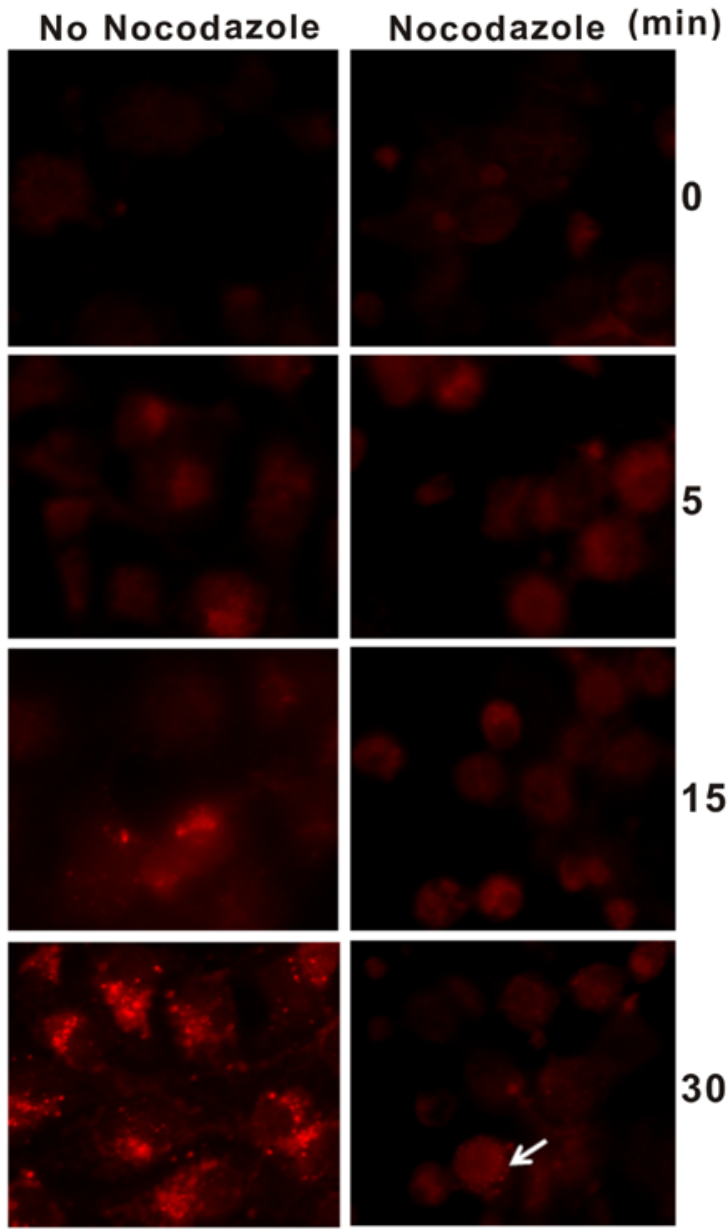

Figure 1. Endocytosis of EGF-EGFR complexes. After COS7 cells were treated for $24 \mathrm{~h}$ with or without nocodazole and then treated with TR-EGF for 5, 15 and $30 \mathrm{~min}$, the cells were fixed with methanol and acetone for 10 min at $-20^{\circ}$ followed by observation of the endosomes in COS7 cells using a fluorescence microscope. The arrow indicates the EGF-EGFR complexes in the plasma membrane.

in asynchronous COS7 cells were significantly increased in the presence of EGF (Fig. 2A). Caveolin expression levels in both the mitotic COS7 and asynchronous COS7 cells were unaffected compared with these levels in the control (Fig. 2B).

EGFR signaling during mitosis. EGFR activation is inhibited during mitosis $(21,22)$. Inhibition of EGFR activity may be beneficial for preventing the activation of signal transduction pathways that promote gene expression to preserve energy needs which are required for mitotic structural changes. After EGFR was stimulated, five important residues (including Y992, Y1048, Y1068, Y1086 and Y1173) were previously found to be phosphorylated; and two residues of which, Y1068 and Y1086, are directly related with Grb2/Shc $(5,6)$. Thus, we detected the two phosphorylation sites. The 1068 and 1086 sites in mitosis were obviously phosphorylated and moreover, their phosphorylation levels were higher than those in the asynchronous COS7 cells (Fig. 3A and B). After EGFR phosphorylation, activated EGFR directly or indirectly recruits various signaling proteins, such as Shc and Grb2 to initiate the signal transduction pathways. Our investigation certified that the expression levels of Grb2 and Shc in the mitotic COS7 cells were unaffected compared with levels in the asynchronous COS7 cells (Fig. 4A-a). However, Grb2 and Shc hardly bound to EGFR in the mitotic COS7 cells (Fig. 4A-b), which directly blocked the downstream protein Ras activity. Since activated Ras mediates numerous cellular functions in different tissues and cell types (1-4), we employed the pull-down assay to detect Ras activity. The results demonstrated that the phosphorylation level of Ras in the mitotic COS7 cells was lower than that in the asynchronous COS7 cells in response to EGF (Fig. 4B), demonstrating the downregulation of Ras activity.

Raf phosphorylation during mitosis. Raf is a downstream protein of Ras. Earlier studies that suggested a potential involvement of ERK in mitotic events reported that Raf-1 was phosphorylated in cells arrested in mitosis with nocodazole (23). In the present study, we investigated Raf phosphorylation. The results demonstrated that Raf phosphorylation by EGF was increased in the mitotic COS7 and asynchronous COS7 cells (Fig. 5A). Except for Ras signaling, PKC activity by PMA can also activate Raf (27). In the present
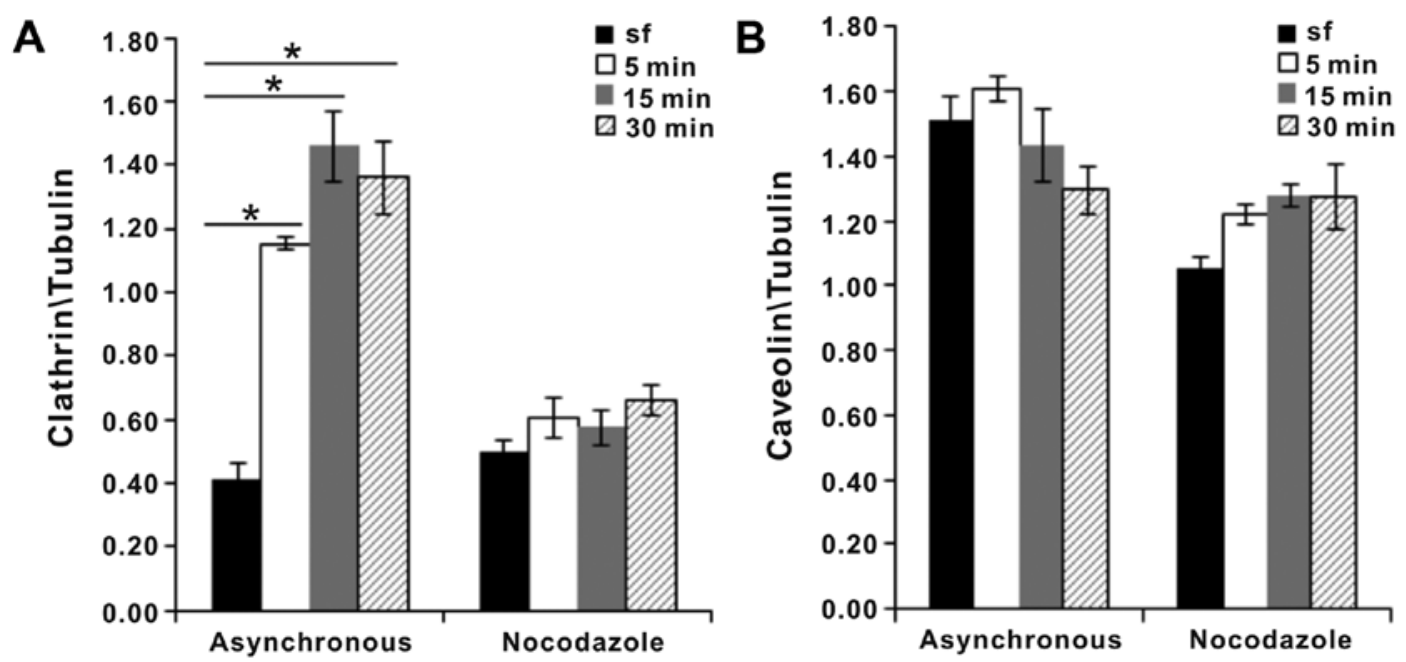

Figure 2. Asynchronous and nocodazole-treated COS7 cells were treated with or without $50 \mathrm{ng} / \mathrm{ml}$ EGF for 5,15 and $30 \mathrm{~min}$. The band intensities of clathrin (A) and caveolin $(\mathrm{B})$ by immunoblotting are shown as determined by ImageJ software. Data are the means \pm standard errors of three separate experiments. $P<0.05$. 
A

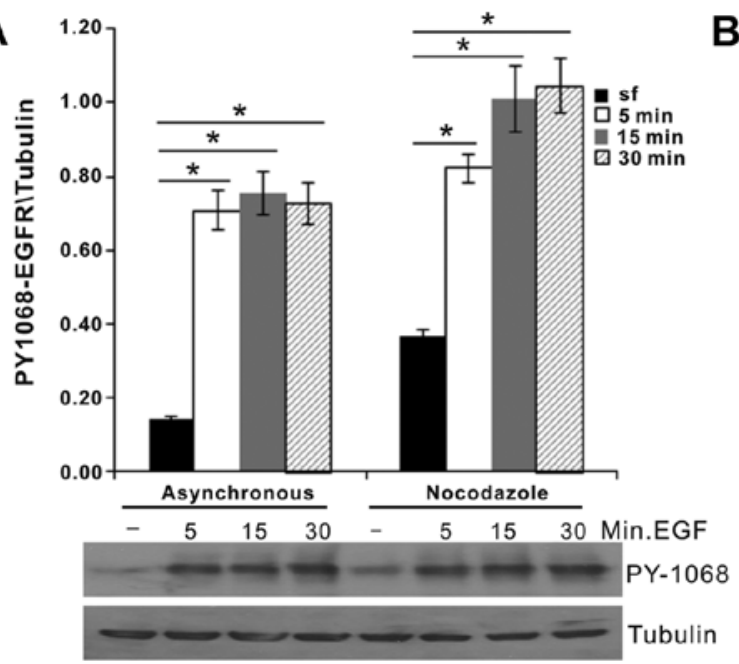

B

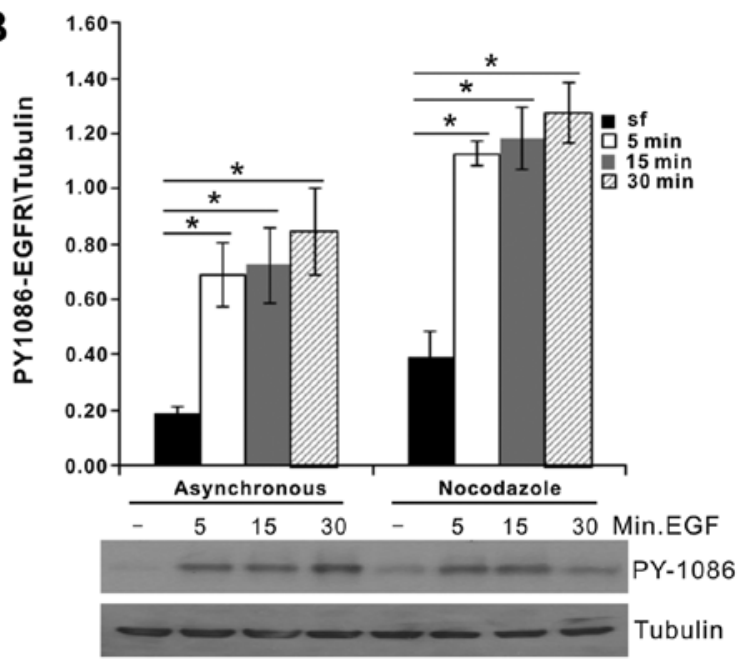

Figure 3. Asynchronous and nocodazole-treated COS7 cells were treated with or without 50 ng/ml EGF for 5 , 15 and 30 min. Y1068 and Y1086 residues of EGFR were detected by immunoblotting, and the band intensities were analyzed by ImageJ software. Data are means \pm standard errors of three separate experiments. ${ }^{*} P<0.05$.
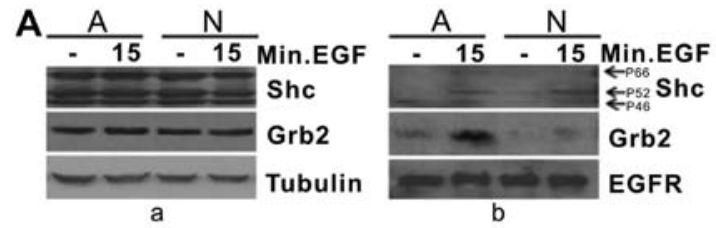

B

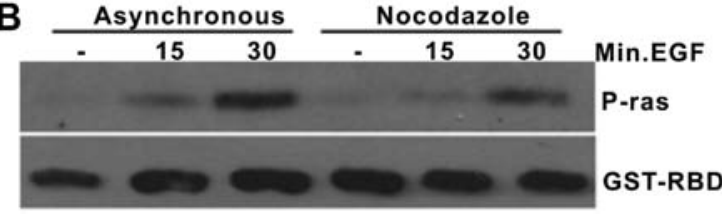

Figure 4. Adaptor analysis. (A) Firstly, asynchronous and nocodazole-treated COS7 cells were treated without or with $50 \mathrm{ng} / \mathrm{ml}$ EGF for $15 \mathrm{~min}$ and were used to analyze the expression of Grb2 and Shc (a). Then, the relationship of EGFR with Grb2 or Shc was detected by the co-immunoprecipitation assays (b). 'A' denotes asynchronous, 'N' denotes nocodazole. (B) The lysate of COS7 cells was incubated with GST-RBD conjugated with glutathione beads, respectively. The glutathione beads were then subjected to immunoblot analysis with a mouse anti-Ras antibody. GST-RBD was stained by a mouse anti-GST antibody to show equal loading.

study, we found that PMA increased the phosphorylation levels of Raf in the mitotic COS7 cells (Fig. 5B), particularly a significant difference was noted in the PMA-treated mitotic COS7 cells for 5 min compared with the PMA-untreated mitotic COS7 cells $(\mathrm{P}<0.05)$. After EGFR activity was inhibited by AG1478, a potent and specific inhibitor of EGF receptor tyrosine kinase activity, EGF-induced phosphorylation of Raf- 1 in the asynchronous and mitotic COS7 cells was significantly blocked (Fig. 5C). These results suggest that Raf phosphorylation is closely associated with EGFR signaling.

Inhibition of MEK and ERK activity during mitosis. Activated Raf phosphorylates and activates MEK and the latter in turn activates ERK. Due to the changes in signaling proteins in the Ras signaling pathway, the expression levels of downstream proteins are possibly affected. To explore the mechanisms involved in Ras signaling in mitosis, the activation of MEK
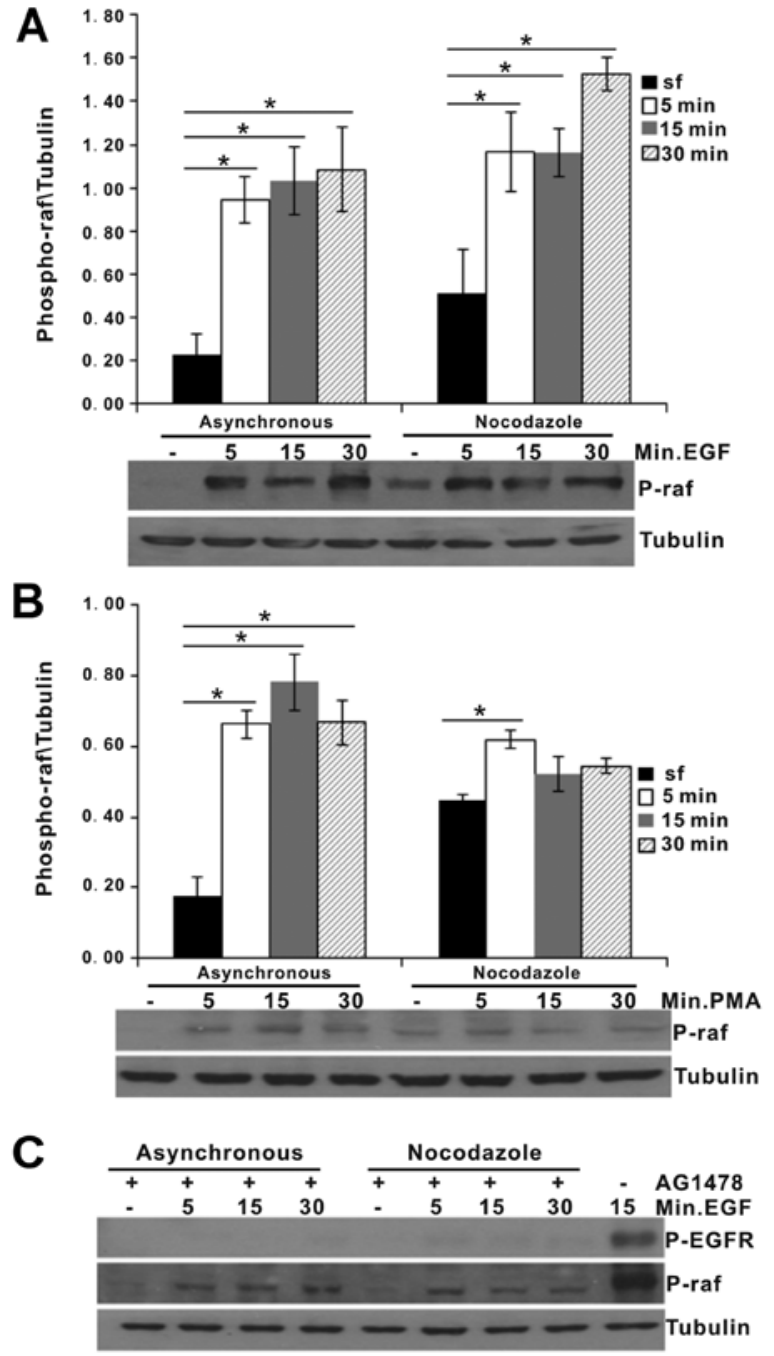

Figure 5. Activation of Raf. Asynchronous and nocodazole-treated COS7 cells were treated with or without $50 \mathrm{ng} / \mathrm{ml}$ EGF (A) or $1 \mu \mathrm{M}$ PMA (B) for 5, 15 and $30 \mathrm{~min}$. Asynchronous and mitotic COS7 cells were treated without

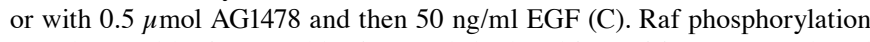
was detected by immunoblotting, and the band intensities were analyzed by Image $J$ software. Data are the means \pm standard errors of three separate experiments. ${ }^{*} P<0.05$. 

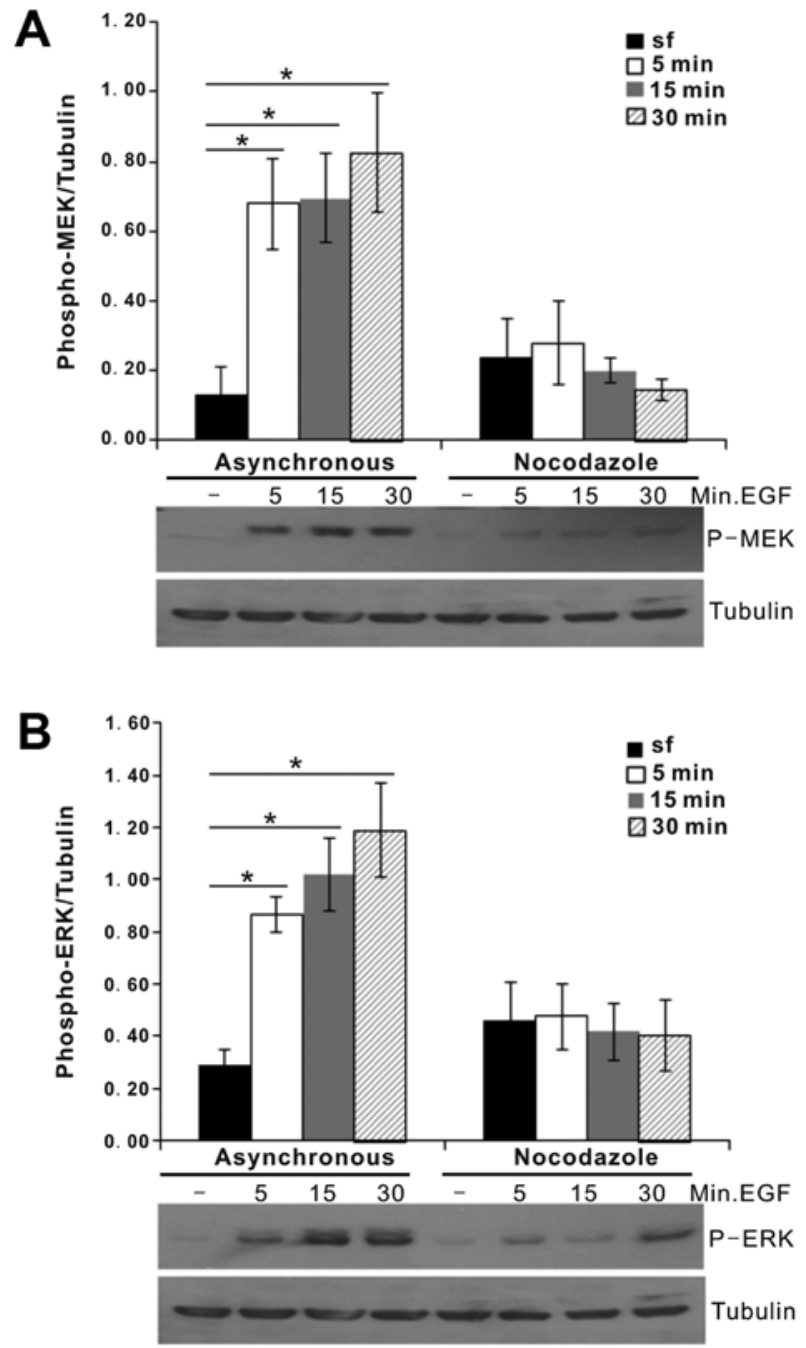

Figure 6. Asynchronous and nocodazole-treated COS7 cells were treated with or without $50 \mathrm{ng} / \mathrm{ml}$ EGF for 5, 15 and $30 \mathrm{~min}$. The phosphorylation levels of MEK and ERK were detected by immunoblotting, and the band intensities of phosphorylated MEK (A) and phosphorylated ERK (B) were analyzed by ImageJ software. Data are the means \pm standard errors of three separate experiments. ${ }^{*} P<0.05$.

and ERK in mitosis and interphase was compared. As expected, the phosphorylation of MEK and ERK in the mitotic COS7 cells in response to EGF was almost completely inhibited; the phosphorylation levels of which were less than that in the asynchronous COS7 cells in the presence of EGF (Fig. 6A and B).

\section{Discussion}

In the present study, we described reduced endosomes of EGF-EGFR during mitosis. The activities of Ras, MEK and ERK by EGF were blocked in mitosis. In addition, we found that the Raf activity in nocodazole-treated COS7 cells was inhibited by AG1478. Grb2 and Shc hardly bound activated EGFR in mitosis. The above results suggest that the molecular mechanism of the Ras-ERK pathway is inhibited in mitotic COS7 cells.

Previous findings indicated that the majority of EGFR without EGF stimulation is concentrated in caveolae via some localization signals (28-31). In response to EGF stimulation,
EGFR quickly exits from caveolae/rafts and undergoes signal transduction and endocytosis (29). In the present study, after COS7 cells were treated for $15 \mathrm{~min}$ by TR-EGF, many endosomes were found in the asynchronous COS7 cells, which suggests that COS7 cells permit the endocytosis of EGF-EGFR complexes and do not quickly degrade these endosomes since internalized EGF and EGFR complexes are targeted to lysosomes for degrading them (32). However, we found that there were few endosomes in the nocodazole-treated COS7 cells, which is possibly related to the physiology of mitotic cells. In mitosis, the membrane traffic is inhibited and EGFR are not dimerized (20), blocking the endocytosis of EGF-EGFR complexes. Another possible reason is that endosomes in mitotic cells are rapidly degraded. To further analyze the endocytosis of EGF-EGFR complexes, we detected clathrin and caveolin which are two important proteins involved in the endocytosis pathway. Generally, the plasma membrane receptor tyrosine kinases (RTKs) are endocytosed through clathrin-dependent and clathrin-independent pathways. We found that clathrin expression in the mitotic COS7 cells was inhibited whereas caveolin expression in mitosis was no different compared with that in asynchronous COS7 cells, demonstrating that the clathrin-dependent pathway is blocked in mitosis (33). Previous research demonstrated that EGFR endocytosed by the clathrin-independent/caveolin pathway is targeted to lysosomes while the EGFR endocytosed by the clathrin-dependent pathway is possibly associated with increased signaling (34). Therefore, inhibition of the clathrin-dependent pathway in mitosis is beneficial to mitotic COS7 cells which will preserve the high energy requirements needed for the dynamic structural changes that are occurring at this time of the cell cycle.

Accumulated evidence suggests that EGFR kinase activation causes auto-phosphorylation of several tyrosine residues within the C-terminal domain. These phosphorylated residues serve as docking sites to recruit downstream signaling proteins and adaptor/accessory proteins containing $\mathrm{SH} 2$ or PTB domains $(35,36)$. EGF-stimulated Ras activation is subjected to two phosphorylated residues such as Y1068 and Y1086 sites $(5,6)$. We investigated that Y1068 and Y1086 residues were obviously phosphorylated in mitosis. It is shown that these residues are unaffected by the inhibition of endocytosis in mitosis. Due to the important roles of the two sites to the Ras-ERK pathway, their activation possibly initiates the Ras-ERK pathway.

$\mathrm{Grb} 2$ is an adaptor protein that contains one $\mathrm{SH} 2$ and two SH3 domains. Grb2 SH2 domain binds to RTKs either directly or indirectly through another adaptor protein, Shc. Grb2 SH3 domains interact with son-of sevenless (Sos). Recruitment of Grb2/Sos to the plasma membrane results in the activation of Ras and the subsequent activation of the Raf-MEK-ERK signaling pathway $(7,8)$. After COS7 cells were collected, we analyzed the interaction of Grb2 and Shc with activated EGFR. The results demonstrated that Grb2 and Shc in the mitotic COS7 cells could not powerfully bind to EGFR compared with that in the asynchronous COS7 cells. It is indicated that EGFR signal transduction possibly is blocked. Due to Grb2 and/or Shc unbinding activated EGFR, Grb2 could not carry Sos to reach the plasma membrane, inhibiting Ras phosphorylation. Then, our results demonstrated that Ras activation was blocked in mitosis, enclosing the Ras-ERK signaling pathway. 
Next, Raf, an important downstream protein of Ras, was detected. Raf is composed of three conservation regions, CR1, CR2 and CR3 (37). The initial process of Raf activation involves the interaction of active GTP-bound Ras with the Ras binding domain (RBD) and the cysteine rich domain of CR1, and subsequent recruitment of Raf to the membrane for further activation $(37,38)$. A previous study demonstrated that Raf in nocodazole-treated COS7 cells could be activated (39). Since Ras in mitosis was inhibited, why was Raf still activated? To answer the question, we collected the mitotic COS7 cells and detected Raf phosphorylation. Treatment with EGF activated Raf in mitosis, and PMA also increased the phosphorylation level of Raf in mitosis. These results suggest that Raf is possibly affected by more than one signaling pathway. Except for Ras, there are other signaling proteins affecting Raf activity in mitotic cells (27). Therefore, although Ras is inhibited in mitosis, Raf possibly is activated by other signaling proteins. Yet, Raf activity is greatly related to EGF stimulation. To confirm the event, we employed AG1478, a special inhibitor of EGFR activity, to treat COS7 cells. The results demonstrated that Raf phosphorylation was blocked by AG1478 in the asynchronous and mitotic COS7 cells, which supports our hypothesis. However, the function of Raf activity in mitosis needs to be further studied.

Activated Raf phosphorylates and activates mitogen-activated protein kinase kinase (MEK) (40-42). MEK in turn phosphorylates ERK via phosphorylation of a Thr-Glu-Tyr motif in the activation loop $(43,44)$. Since Raf in mitosis is activated and MEK/ERK are its downstream proteins, we collected and analyzed the phosphorylation of MEK/ERK. Our finding demonstrated that MEK/ERK phosphorylation by EGF in mitosis was inhibited compared with that in the asynchronous COS7 cells. These results suggest that activated Raf in mitosis possibly loses the ability to stimulate downstream signaling proteins, consistent with the results of Laird et al (45).

In conclusion, the alteration of EGFR endocytosis in mitotic COS7 cells affects cell signaling. The activities of signaling proteins including Ras, MEK and ERK were significantly decreased in the mitotic COS7 cells. Due to the difficulty in the transduction of EGF signaling by EGFR or Raf to downstream proteins, Ras-ERK pathway in mitotic COS7 cells is blocked in dual pressures of signal transduction inhibition.

\section{Acknowledgements}

The present study was supported by the 'National Natural Science Foundation of China (31272409)' and the 'Science Foundation of Shaanxi Province of China (2013KTZB02-02-03)'.

\section{References}

1. Cha DS, Datla US, Hollis SE, Kimble J and Lee MH: The Ras-ERK MAPK regulatory network controls dedifferentiation in Caenorhabditis elegans germline. Biochim Biophys Acta 1823: 1847-1855, 2012.

2. Choi C and Helfman DM: The Ras-ERK pathway modulates cytoskeleton organization, cell motility and lung metastasis signature genes in MDA-MB-231 LM2. Oncogene 33: 3668-3676, 2014.

3. Nishida Y: Function of Raf/MAP kinase cascade in the regulation of cellular proliferation and differentiation. Tanpakushitsu Kakusan Koso 41 (Suppl 12): S1673-S1679, 1996.
4. Yoon S and Seger R: The extracellular signal-regulated kinase: Multiple substrates regulate diverse cellular functions. Growth Factors 24: 21-44, 2006.

5. Batzer AG, Rotin D, Ureña JM, Skolnik EY and Schlessinger J: Hierarchy of binding sites for Grb2 and Shc on the epidermal growth factor receptor. Mol Cell Biol 14: 5192-5201, 1994.

6. Sasaoka T, Langlois WJ, Leitner JW, Draznin B and Olefsky JM: The signaling pathway coupling epidermal growth factor receptors to activation of p21 ${ }^{\text {ras }}$. J Biol Chem 269: 32621-32625, 1994.

7. Kolch W: Meaningful relationships: The regulation of the Ras/Raf/MEK/ERK pathway by protein interactions. Biochem J 351: 289-305, 2000.

8. Roberts PJ and Der CJ: Targeting the Raf-MEK-ERK mitogenactivated protein kinase cascade for the treatment of cancer. Oncogene 26: 3291-3310, 2007.

9. Higashi N, Kunimoto H, Kaneko S, Sasaki T, Ishii M, Kojima $\mathrm{H}$ and Nakajima K: Cytoplasmic c-Fos induced by the YXXQ-derived STAT3 signal requires the co-operative MEK/ERK signal for its nuclear translocation. Genes Cells 9: 233-242, 2004

10. Manimala NJ, Frost CD, Lane ml, Higuera M, Beg R and Vesely DL: Cardiac hormones target nuclear oncogenes c-Fos and c-Jun in carcinoma cells. Eur J Clin Invest 43: 1156-1162, 2013.

11. Lavoie JN, L'Allemain G, Brunet A, Müller R and Pouysségur J: Cyclin D1 expression is regulated positively by the p42/p44 ${ }^{\text {MAPK }}$ and negatively by the $\mathrm{p} 38 / \mathrm{HOG}^{M A P K}$ pathway. J Biol Chem 271: 20608-20616, 1996.

12. Weber JD, Raben DM, Phillips PJ and Baldassare JJ: Sustained activation of extracellular-signal-regulated kinase 1 (ERK1) is required for the continued expression of cyclin D1 in G1 phase. Biochem J 326: 61-68, 1997.

13. Berlin RD, Oliver JM and Walter RJ: Surface functions during Mitosis I: Phagocytosis, pinocytosis and mobility of surfacebound Con A. Cell 15: 327-341, 1978.

14. Berlin RD and Oliver JM: Surface functions during mitosis. II. Quantitation of pinocytosis and kinetic characterization of the mitotic cycle with a new fluorescence technique. J Cell Biol 85: 660-671, 1980.

15. Kreiner T and Moore HP: Membrane traffic between secretory compartments is differentially affected during mitosis. Cell Regul 1: 415-424, 1990.

16. Sager PR, Brown PA and Berlin RD: Analysis of transferrin recycling in mitotic and interphase HeLa cells by quantitative fluorescence microscopy. Cell 39: 275-282, 1984.

17. Warren G, Featherstone C, Griffiths G and Burke B: Newly synthesized $G$ protein of vesicular stomatitis virus is not transported to the cell surface during mitosis. J Cell Biol 97: $1623-1628,1983$.

18. Hayne C, Xiang X and Luo Z: MEK inhibition and phosphorylation of serine 4 on B23 are two coincident events in mitosis. Biochem Biophys Res Commun 321: 675-680, 2004.

19. Margadant C, Cremers L, Sonnenberg A and Boonstra J: MAPK uncouples cell cycle progression from cell spreading and cytoskeletal organization in cycling cells. Cell Mol Life Sci 70: 293-307, 2013.

20. Kiyokawa N, Lee EK, Karunagaran D, Lin SY and Hung MC: Mitosis-specific negative regulation of epidermal growth factor receptor, triggered by a decrease in ligand binding and dimerization, can be overcome by overexpression of receptor. J Biol Chem 272: 18656-18665, 1997.

21. Newberry EP and Pike LJ: Cell-cycle-dependent modulation of EGF-receptor-mediated signaling. Biochem Biophys Res Commun 208: 253-259, 1995.

22. Klein S, Kaszkin M, Barth H and Kinzel V: Signal transduction through epidermal growth factor receptor is altered in $\mathrm{HeLa}$ monolayer cells during mitosis. Biochem J 322: 937-946, 1997.

23. Laird AD, Taylor SJ, Oberst M and Shalloway D: Raf-1 is activated during mitosis. J Biol Chem 270: 26742-26745, 1995.

24. Herrmann C, Martin GA and Wittinghofer A: Quantitative analysis of the complex between $\mathrm{p} 21^{\text {ras }}$ and the Ras-binding domain of the human Raf-1 protein kinase. J Biol Chem 270: 2901-2905, 1995.

25. Cochet C, Kashles O, Chambaz EM, Borrello I, King CR and Schlessinger J: Demonstration of epidermal growth factorinduced receptor dimerization in living cells using a chemical covalent cross-linking agent. J Biol Chem 263: 3290-3295, 1988.

26. Gamett DC, Pearson G, Cerione RA and Friedberg I: Secondary dimerization between members of the epidermal growth factor receptor family. J Biol Chem 272: 12052-12056, 1997. 
27. Hyde R, Corkins ME, Somers GA and Hart AC: PKC-1 acts with the ERK MAPK signaling pathway to regulate Caenorhabditis elegans mechanosensory response. Genes Brain Behav 10 : 286-298, 2011

28. Couet J, Sargiacomo M and Lisanti MP: Interaction of a receptor tyrosine kinase, EGF-R, with caveolins. Caveolin binding negatively regulates tyrosine and serine/threonine kinase activities. J Biol Chem 272: 30429-30438, 1997.

29. Mineo C, Gill GN and Anderson RG: Regulated migration of epidermal growth factor receptor from caveolae. J Biol Chem 274: 30636-30643, 1999.

30. Smart EJ, Ying YS, Mineo C and Anderson RG: A detergent-free method for purifying caveolae membrane from tissue culture cells. Proc Natl Acad Sci USA 92: 10104-10108, 1995.

31. Yamabhai M and Anderson RG: Second cysteine-rich region of epidermal growth factor receptor contains targeting information for caveolae/rafts. J Biol Chem 277: 24843-24846, 2002.

32. Carpenter G: Receptors for epidermal growth factor and other polypeptide mitogens. Annu Rev Biochem 56: 881-914, 1987.

33. Fielding AB, Willox AK, OkekeEand Royle SJ:Clathrin-mediated endocytosis is inhibited during mitosis. Proc Natl Acad Sci USA 109: 6572-6577, 2012.

34. Aguilar RC and Wendland B: Endocytosis of membrane receptors: Two pathways are better than one. Proc Natl Acad Sci USA 102: 2679-2680, 2005.

35. Jorissen RN, Walker F, Pouliot N, Garrett TP, Ward CW and Burgess AW: Epidermal growth factor receptor: Mechanisms of activation and signalling. Exp Cell Res 284: 31-53, 2003.

36. Pawson T: Specificity in signal transduction: From phosphotyrosine-SH2 domain interactions to complex cellular systems. Cell 116: 191-203, 2004.
37. Morrison DK and Cutler RE Jr: The complexity of Raf-1 regulation. Curr Opin Cell Biol 9: 174-179, 1997.

38. Vojtek AB, Hollenberg SM and Cooper JA: Mammalian Ras interacts directly with the serine/threonine kinase Raf. Cell 74: 205-214, 1993.

39. Dangi S and Shapiro P: Cdc2-mediated inhibition of epidermal growth factor activation of the extracellular signal-regulated kinase pathway during mitosis. J Biol Chem 280: 24524-24531, 2005.

40. Dent P, Haser W, Haystead TA, Vincent LA, Roberts TM and Sturgill TW: Activation of mitogen-activated protein kinase kinase by v-Raf in NIH 3 T3 cells and in vitro. Science 257: 1404-1407, 1992.

41. Howe LR, Leevers SJ, Gómez N, Nakielny S, Cohen P and Marshall CJ: Activation of the MAP kinase pathway by the protein kinase raf. Cell 71: 335-342, 1992

42. Kyriakis JM, App H, Zhang XF, Banerjee P, Brautigan DL, Rapp UR and Avruch J: Raf-1 activates MAP kinase-kinase. Nature 358: 417-421, 1992.

43. Lange-Carter CA, Pleiman CM, Gardner AM, Blumer KJ and Johnson GL: A divergence in the MAP kinase regulatory network defined by MEK kinase and Raf. Science 260: 315-319, 1993.

44. Moodie SA, Willumsen BM, Weber MJ and Wolfman A: Complexes of Ras.GTP with Raf-1 and mitogen-activated protein kinase kinase. Science 260: 1658-1661, 1993.

45. Laird AD, Morrison DK and Shalloway D: Characterization of Raf-1 activation in mitosis. J Biol Chem 274: 4430-4439, 1999. 